Case Report

\title{
Assessment of Static Steadiness and Dynamic Stability at Various Stages of Healing of a Grade 2 Medial Collateral Ligament Tear: A Case Report
}

\author{
Kaycee Glattke*, Michael Smith, Toshiki Tsuchiya, Jakob Wells and Thurmon Lockhart *D \\ School of Biological and Health Systems Engineering, Arizona State University, Tempe, AZ 85281, USA; \\ mdsmit25@asu.edu (M.S.); ttsuchi2@asu.edu (T.T.); jgwells2@asu.edu (J.W.) \\ * Correspondence: kglattke@asu.edu (K.G.); thurmon.lockhart@asu.edu (T.L.)
}

Citation: Glattke, K.; Smith, M.; Tsuchiya, T.; Wells, J.; Lockhart, T. Assessment of Static Steadiness and Dynamic Stability at Various Stages of Healing of a Grade 2 Medial Collateral Ligament Tear: A Case Report. Sci 2021, 3, 21. https:// doi.org/10.3390/sci3020021

Academic Editor: Claus Jacob

Received: 26 September 2019

Accepted: 31 March 2021

Published: 7 April 202

Publisher's Note: MDPI stays neutral with regard to jurisdictional claims in published maps and institutional affiliations.

Copyright: (c) 2021 by the authors. Licensee MDPI, Basel, Switzerland This article is an open access article distributed under the terms and conditions of the Creative Commons Attribution (CC BY) license (https:// creativecommons.org/licenses/by/ $4.0 /)$.

\begin{abstract}
Injuries to ligaments of the knee are extremely common among athletes who participate in high-risk sports or any sport that requires frequent cutting motions, jumping, or contact. In order to determine the best way to heal these injuries, it is important to understand not just the pathology of the injury but also the biomechanical factors that are affected, including stability and steadiness. While many studies have been conducted to examine the stability of healthy knees, there is little to no existing literature on stability of knees afflicted by injury. In order to surpass this obstacle, static steadiness and dynamic stability data were collected using the Lockhart Monitor phone application and Xsens accelerometers, respectively, both before and after completion of a course of physical therapy in a subject with a grade 2 medial collateral ligament (MCL) tear. These results were then used to determine the degree to which the prescribed physical therapy protocol was effective in healing the MCL, which can be useful for adjusting the individual protocol for future conservative treatment and management of the injury.
\end{abstract}

Keywords: biomechanics; dynamic stability; inertial measurement unit; medial collateral ligament; static stability

\section{Introduction}

The medial collateral ligament $(\mathrm{MCL})$ is one of the most frequently injured structures of the knee, especially by athletes who participate in sports with copious amounts of valgus knee loading [1]. Fortunately, most people who sustain an injury to the MCL can achieve pre-injury activity levels with conservative non-surgical treatment; however, it is not uncommon for other structures to be damaged in addition to the MCL as a result of traumatic injury to the knee. If the injury is isolated to the MCL, the most common forms of conservative treatment include stabilization and rehabilitation, and if instability persists, then surgical reconstruction or repair may be required [2].

\subsection{Anatomy}

The anatomy of the knee is relatively complex and consists of many soft tissue and bony structures located in the joint space between the femur and tibia. The four main ligaments that serve as primary stabilizers of the knee include the anterior cruciate ligament (ACL), MCL, lateral collateral ligament (LCL), and the posterior cruciate ligament (PCL). The ACL and PCL are intra-articular ligaments within the joint capsule of the knee that restrict anterior and posterior movement of the tibia with respect to the femur and the MCL and LCL stabilize the knee on both of its sides. In addition to these stabilizing ligaments, there are two cartilaginous structures that act as shock absorbers for axial stresses between the femur and tibia: the medial and lateral menisci [3]. The primary biomechanical function of the medial structures of the knee is to provide stability against valgus stress as well as 
both internal and external rotation. The superficial MCL acts as the primary stabilizer to valgus stress and the deep MCL acts as the secondary stabilizer [2].

\subsection{Etiology and Clinical Presentation}

Injuries to the MCL can occur as a result of both contact and non-contact activities, usually during sports. Common examples of injuries include lateral blows to the knee which result in extreme valgus stress as well as external rotation of the foot while the knee is flexed [2].

Upon examination by a clinician, patients typically present with some sort of traumatic injury history to the medial portion of the knee, and most report hearing an auditory "pop" as well as feeling a tearing sensation within the knee upon injury. If a clinician suspects that the MCL has been torn, he or she can perform a valgus stress test, where the leg is extended and stress is applied medially to the knee. The clinician can then assess how much laxity is present in the affected joint, and can prescribe appropriate imaging, such as an MRI, to diagnose the severity of the injury and determine which, if any, other structures are involved.

Injuries to the MCL are graded on a scale of 1-3; grade 1 tears involve very few fibers $(0-10 \%)$ and generally have no associated instability, grade 2 tears could involve anywhere from 10 to $90 \%$ of the fibers, and grade 3 tears are considered a full tear with little to no fibers remaining intact [4]. The superficial MCL also has an abundant vascular supply, which contributes to its high response rate to conservative treatment [4].

\section{Materials and Methods}

\subsection{Aim}

The purpose of this experimental study was to observe the effects of a single course of conservative rehabilitation of an MCL tear on the static and dynamic stability of an afflicted subject. Both forms of stability were quantified before and after a five-week physical therapy regimen in a single subject with an isolated grade 2 MCL tear.

\subsection{Subject}

Eligible subjects for this study included those between the ages of 18 and 30 that had recently been diagnosed with an isolated grade $2 \mathrm{MCL}$ tear or sprain and who were able to comply with the study procedures below. Exclusion criteria include those with a previous history of injury or surgery to either knee and those with a history of other musculoskeletal disorders affecting gait and balance. Subjects were recruited from a population of graduate students and a total of one subject was enrolled in this study, who had recently experienced a grade 2 MCL tear in the left knee as a result of an external rotation of the foot while the knee was flexed. Informed consent was obtained by the subject after it was determined that they met all inclusion criteria. Following injury, the subject had not used crutches or any other assistive devices to aid in their gait.

\subsection{Apparatuses}

Two devices were used to measure static and dynamic stability. For the static portion, an iOS application named "Lockhart Monitor" (created by Thurmon Lockhart, PhD; Arizona State University) was used to measure both sway area and sway velocity. The application is freely available to the public for download on the Apple App Store and was used on an iPhone 7 (Vendor: Apple, Inc.; Cupertino, CA, USA) that was secured to an elastic strap placed around the subject's waist. This location was chosen as it is typical for gait research to be conducted with wearable sensors located as close to the subject's center of mass as possible, which is at the lower back or, specifically, the fifth lumbar vertebrae (L5) [5]. The Lockhart Monitor application has been validated in literature for use in various clinical populations [6]. For the dynamic portion, an Xsens MTw Awinda motion trackers (Vendor: Clearpath Robotics; Waterloo, ON, USA) were used at four anatomical landmarks on the body [7]. One sensor was attached to the sacrum, one was attached 
to the trunk at the midpoint of the sternum, and one was attached on the lateral side of each shank directly above the lateral malleolus. The subject walked around a track while a wireless receiver for the Xsens MTw Awinda motion trackers was carried by an investigator nearby to collect data at a sampling frequency of $120 \mathrm{~Hz}$.

\subsection{Procedure}

\section{Baseline Data Collection}

Baseline data were collected approximately two months after the subject sustained their injury and, additionally, one week before beginning their rehabilitation. The subject underwent five weeks of supervised physical therapy under the care of a licensed physical therapist and then returned for their second and final data collection session. At the baseline data collection session, the subject first completed four trials of static stability measurements using the Lockhart Monitor application on the iPhone. For these trials, the subject stood on both legs for $60 \mathrm{~s}$ with the iPhone attached to an elastic band around their waist. The subject stood with their feet at hip-width apart and with their feet pointed straight ahead while wearing shoes. Two of these trials were completed with the eyes open and two were completed with the eyes closed. For the eyes-open trials, the subject was instructed to focus on a single point on the wall across from where they stood. Then, the subject completed one trial of single-leg stability for each side while standing on only one leg and with eyes open for a duration of $30 \mathrm{~s}$. Immediately after, the subject walked around a track $\left(100^{\prime} \times 50^{\prime}\right)$ for six minutes to collect dynamic stability data using the Xsens MTw Awinda sensors. The subject was instructed to walk at a self-selected pace and to keep the pace as constant as possible throughout the entire duration of the test. Data from the subject walking along the straight-line portion of the track was kept for analysis, while data from walking around the corners was discarded. These data were isolated by searching throughout the collected data and deleting data beginning with any observed anomalies that were regularly repeated throughout. Data were de-identified and stored in a secure cloud-based storage system and only members of the study team were granted access to the data.

\section{Physical Therapy}

After the baseline set of measurements, the subject completed five weeks of physical therapy with two visits per week. At the first physical therapy visit, the subject underwent a complete physical evaluation of the injured limb. Findings of this evaluation included muscular deficits in the quadriceps, hamstrings, and gluteal muscles of both legs with markedly decreased muscular function of the injured leg compared to the non-injured leg. No deficits in range of motion were noted and gait patterns appeared normal. Each session lasted approximately $45 \mathrm{~min}$ and consisted of a variety of lower extremity exercises.

These exercises target the major muscle groups of the lower extremities, which act as secondary stabilizers to the knee joint. As these muscle groups are strengthened, stress is relieved from the ligaments of the knee joint during any activities in which it is used. This is particularly important when any of the ligaments are injured as unnecessary stress or strain on the injured ligament should be avoided in order to facilitate healing. These muscle groups also play an integral role in static and dynamic stability as any sort of balance activity utilizes these muscles in the lower extremity. Muscular deficits in the lower extremities can result in poor stability and balance; thus, strengthening these muscles would, in theory, lead to better postural control and dynamic stability during gait.

The first two weeks of physical therapy consisted of single-plane exercises aimed at strengthening individual muscle groups: the quadriceps, hip and gluteal muscles, and the calves. First, neuromuscular electric stimulation (NMES) was applied to the quadriceps of the afflicted leg for using a Chattanooga Vectra Genisys Model 2761 (DJO, LLC; Lewisville, TX, USA). NMES is often used in rehabilitation for various knee injuries with the goal of increasing muscle fiber recruitment of the quadriceps to facilitate and improve its activation [8]. For this particular subject, the NMES settings used were Russian electric stimulation, single channel, sinusoidal waveform delivered in a series of pulses. The 
subject completed quadriceps sets during each pulse of electric stimulation for ten minutes. Following this, the subject completed the following exercise regimen with the aim of improving activation, strength, and endurance in muscles of the lower extremities:

- Quadriceps strengthening

- Straight leg raises: 2 sets of 10 repetitions

- Long arc quads: 2 sets of 10 repetitions

- Terminal knee extension (TKE): 2 sets of 10 repetitions

- Hip and glute strengthening

- Double-leg bridges: 2 sets of 10 repetitions

- Side-lying hip external rotation (clamshells): 2 sets of 10 repetitions

- Side-lying hip abduction: 2 sets of 10 repetitions

- Calf strengthening

- Heel raises: 2 sets of 10 repetitions

As the subject progressed from active range of motion exercises to resisted range of motion exercises, exercise intensity was increased by the addition of resistance with TheraBand as tolerated. This progression was allowed based on rate of perceived exertion (RPE); that is, when the subject had determined that the exercises performed were light or very light activity, resistance would be added or increased until the activity was considered moderate or vigorous.

The final three weeks of physical therapy progressed from the isolated, single-plane exercises to multiplanar functional exercises that utilized combinations of the lower extremity muscle groups. These exercises were as follows:

Standing hip four-way with TheraBand: 2 sets of 10 repetitions

Mini wall squats: 2 sets of 10 repetitions

Lateral band walks: 2 sets of 10 repetitions

Monster walks: 2 sets of 10 repetitions

- Leg press in pain-free range of motion ( $35 \mathrm{lbs})$ : 2 sets of 10 repetitions

The subject also utilized cryotherapy, bracing with a commercially available knee brace, and kinesiotaping, as needed, at the recommendation of their physical therapist.

\section{Follow-Up Data Collection}

Following the completion of five weeks of supervised physical therapy, the subject returned for their second and final set of data collection. The protocol was identical to the initial data collection performed pre-therapy. At the second data collection session, the subject's knee was taped with kinesiotape as part of their individualized physical therapy protocol.

\section{Results}

\subsection{Postural Stability}

Romberg ratio $(\mathrm{QR})$ is a method of analyzing postural stability using sway area and sway velocity. This ratio is defined as eyes closed divided by eyes open (EC/EO) and can be calculated using both sway area and sway velocity. Sway area and sway velocity were collected using the Lockhart Monitor application, which utilizes the inertial measurement unit within an iPhone to measure the area and velocity of the estimated center of pressure (COP) relative to where the phone is located. Romberg ratio quantifies the reliance of postural stability on visual feedback [9].

The data in Table 1 alone show little difference between the trials before and after physical therapy, demonstrating that the postural stability's reliance on visual feedback did not change significantly before and after physical therapy. 
Table 1. Average Romberg Ratio (QR).

\begin{tabular}{cccc}
\hline \multicolumn{2}{c}{ Pre-Physical Therapy } & \multicolumn{2}{c}{ Post-Physical Therapy } \\
\hline Velocity & Area & Velocity & Area \\
\hline 0.99 & 0.74 & 1.01 & 0.96 \\
\hline
\end{tabular}

Alternate Romberg ratio (Alternate $\mathrm{QR}$ ) is defined as ((eyes closed - eyes open)/(eyes closed + eyes open) $) \times 100$ and can be calculated using both sway velocity and sway area. Alternate Romberg ratio is used to decrease the inter-trial variance that could potentially occur during consecutive trials [9]. Values of Alternate QR that are close to zero or are negative demonstrate less reliance on visual feedback to maintain postural control.

The data in Table 2 demonstrate an increase in reliance on visual feedback for sway velocity, with a decrease in reliance on visual feedback for sway area. This shows that after physical therapy, the subject is now more apt to use their visual system over input from other areas of the feedback control loop. This indicates a decreased reliance on proprioceptive feedback and overall improvement in the subject's steadiness abilities.

Table 2. Alternate Romberg Ratio (QR).

\begin{tabular}{cccc}
\hline \multicolumn{2}{c}{ Pre-Physical Therapy } & \multicolumn{2}{c}{ Post-Physical Therapy } \\
\hline Velocity & Area & Velocity & Area \\
\hline 4.56 & 26.19 & 8.84 & 19.1 \\
\hline
\end{tabular}

Table 3 shows single-leg postural steadiness for both the affected (left) and unaffected (right) legs. The steadiness has increased after completion of physical therapy, indicating an increase in the function of the muscles involved in the single-leg postural steadiness test, such as the hamstrings. The subject demonstrates a greater ability to balance on each individual leg when compared to before the physical therapy occurred. To understand how the subject is progressing with regards to the injury, it is important to analyze the ratio of the steadiness as a function of both legs.

Table 3. Single-Leg Postural Steadiness.

\begin{tabular}{ccccccccc}
\hline & \multicolumn{2}{c}{ Affected Leg } & \multicolumn{3}{c}{ Unaffected Leg } \\
\hline \multicolumn{2}{c}{$\begin{array}{c}\text { Pre-Physical } \\
\text { Therapy }\end{array}$} & \multicolumn{2}{c}{$\begin{array}{c}\text { Post-Physical } \\
\text { Therapy }\end{array}$} & \multicolumn{2}{c}{$\begin{array}{c}\text { Pre-Physical } \\
\text { Therapy }\end{array}$} & \multicolumn{2}{c}{$\begin{array}{c}\text { Post-Physcial } \\
\text { Therapy }\end{array}$} \\
\hline Velocity & Area & Velocity & Area & Velocity & Area & Velocity & Area \\
\hline 0.595 & 15.433 & 0.496 & 3.569 & 0.583 & 16.029 & 0.326 & 2.000 \\
\hline
\end{tabular}

The data in Table 4 present single-leg postural steadiness expressed as a ratio of the affected leg over the unaffected leg before and after the physical therapy treatments. A ratio of greater than 1 would indicate that the injured leg is stronger than the healthy leg, while a ratio less than 1 would indicate that the healthy leg is the stronger of the two. The data in Table 4 show that this ratio decreased significantly after physical therapy, which would be indicative of the muscles in the affected leg weakening over time. Although individual leg steadiness may have improved, the ratio of the two has not. This could be due to compensation from the healthy leg or significant muscular imbalances between the two legs, which can contribute to an increased risk of reinjury.

Table 4. Single-Leg Postural Steadiness Ratio: Affected/Unaffected Leg.

\begin{tabular}{cccc}
\hline \multicolumn{2}{c}{ Pre-Physical Therapy } & \multicolumn{2}{c}{ Post-Physical Therapy } \\
\hline Velocity & Area & Velocity & Area \\
\hline 1.02 & 1.04 & 0.66 & 0.56 \\
\hline
\end{tabular}


The results from the static postural stability show overall improvement in stability and steadiness in terms of individual legs. However, when comparing the legs to each other, the steadiness or stability of the subject shows a general decrease as a result of the physical therapy. This implies that the physical therapy is not beneficial to the required healing process for this particular patient with regards to stability, and alternative methods of treatment should perhaps be discussed and prescribed.

\subsection{Local Dynamic Stability}

Local dynamic stability was quantified using maximum Lyapunov Exponent, or maxLE [10]. The kinematic measurements taken from four different anatomical landmarks were separately analyzed to compute maxLE. In order to isolate each gait cycle, signals with relatively clean peaks were selected for the analysis. The angular velocity recordings of foot movement in the sagittal plane were used for the right and left shanks. For trunk and sacrum, linear acceleration in the superior-inferior direction was used. MaxLE was calculated every 50 gait cycles and the mean values can be found in Table 5 below. All four anatomical landmarks demonstrated improvement in dynamic stability, which can be seen with the decrease of maxLE from pre-PT to post-PT. Notably, two sample $t$-tests were performed and there was a statistically significant change in the left shank $(p=0.004$, $\alpha=0.05)$, ipsilateral to the location of the injury. In both pre-PT and post-PT, the subject had relatively higher upper body stability, in contrast to the legs.

Table 5. Mean MaxLE.

\begin{tabular}{cccccccc}
\hline \multicolumn{2}{c}{ Right Shank } & \multicolumn{2}{c}{ Left Shank } & \multicolumn{2}{c}{ Trunk } & \multicolumn{2}{c}{ Sacrum } \\
\hline Pre-PT & Post-PT & Pre-PT & Post-PT & Pre-PT & Post-PT & Pre-PT & Post-PT \\
\hline $1.41 \pm 0.15$ & $1.36 \pm 0.11$ & $1.36 \pm 0.10$ & $0.92 \pm 0.30$ & $0.81 \pm 0.03$ & $0.79 \pm 0.07$ & $0.71 \pm 0.07$ & $0.69 \pm 0.06$ \\
\hline
\end{tabular}

\subsection{Stride Duration and Cadence}

The variability in stride duration was studied to assess potential compensation mechanisms contributing to the dynamic stabilization. Stride duration was calculated at the mean stride duration of each straight portion of the track divided by the time over which the subject was walking. The mean stride duration from pre-PT to post-PT decreased with fairly high consistency as seen from the low variance. Additionally, the cadence naturally increased with decrease in stride duration. The root-mean-square of stride duration in both legs decreased from pre-PT to post-PT, which suggests an improvement in neuromotor control of stride duration variability. These data can be seen in Table 6 below. A previous study has shown that the increase in stride frequency (decrease in stride duration) can improve the mediolateral margin of stability [11]. The results may be indicative of such compensatory mechanism present in the subject, but further study would be needed to verify this.

Table 6. Stride Duration.

\begin{tabular}{ccc}
\hline & Pre-PT & Post-PT \\
\hline $\begin{array}{c}\text { Left foot } \\
\text { Mean (seconds) }\end{array}$ & 1.106 & 1.063 \\
\hline Variance (seconds) & 0.001 & 0.006 \\
\hline Root-Mean-Square (seconds) & 1.106 & 1.063 \\
\hline $\begin{array}{c}\text { Right foot } \\
\text { Mean (seconds) }\end{array}$ & 1.105 & 1.063 \\
\hline Variance (seconds) & 0.0009 & 0.0006 \\
\hline Root-Mean-Square (seconds) & 1.106 & 1.063 \\
\hline Mean Cadence (steps) & 109 & 113 \\
\hline
\end{tabular}




\section{Discussion}

There are two distinct possibilities for the outcome of this case study. The first and most optimal outcome is that the subject would have tolerated conservative treatment well and shown improvements in both static and dynamic stability following the fiveweek physical therapy interval. This would indicate that the subject's therapy sessions had improved their postural stability for both walking and standing. The other possible outcome is that the subject's stability would have either worsened or failed to improve with conservative treatment. In this instance, the physical therapy would not be considered effective and other non-conservative methods of treatment would need to be explored. It has been found that, following diagnosis by a licensed orthopedic surgeon, the subject has experienced residual laxity in the MCL as a result of their injury. In this case, they are not as likely to benefit from continued conservative treatment and operative treatment should begin to be considered.

Surgical options for MCL injuries that do not respond to nonoperative treatment include either repair or reconstruction. For example, a surgical technique called internal bracing has recently gained popularity for treatment of MCL injury [12]. This involves the use of biodegradable tacks that re-tension the MCL and strips of biodegradable fibers that reinforce the MCL while it heals. In cases where there are no intact fibers of the MCL remaining following injury, such as in grade 3 tears, surgical reconstruction of the ligament is typically the preferred surgical treatment [13]. Reconstruction of the MCL is usually completed with cadaveric allografts due to the relatively large size of the ligament in order to prevent creating unnecessary deficiencies in healthy tissue by harvesting an autograft [13].

This study is not without its limitations. First and foremost, the power of this study would benefit from the recruitment of more eligible subjects. The small sample size is the result of a very limited timeframe for recruitment as well as a small population of potential subjects to recruit from. Thus, the decision was made for this investigation to be completed as a case study. Next, this study would also have benefitted from the opportunity to collect data at more points in time, such as periodically throughout the physical therapy intervention. It would have been of particular interest to also collect electromyography (EMG) feedback data throughout the trials in order to obtain more information regarding muscular function and activation during the stability tasks; however, the necessary equipment for EMG was not available at the time this study was conducted.

It would have also been beneficial to perform a full gait analysis using a motion capture system and instrumented treadmill. Positional tracking would allow for estimation of the center of mass, which can then be used to calculate the margin of stability, a parameter potentially present in the control mechanism of post-PT walking. It would also be a great addendum to the study to perform another full postural and stability analysis at different points of postoperative recovery. It is also worth noting that the tests in this study should only be performed if they do not cause pain or harm to the subject's afflicted knee.

\section{Conclusions}

Knee injuries are an orthopedic epidemic; they are suffered by people of all ages and activity levels because of the vulnerability of the knee's soft tissue structures. The analysis completed in this study only begins to scratch the surface of biomechanical factors that can be investigated following an injury to the $\mathrm{MCL}$, not to mention the fact that injuries to the other ligaments and structures of the knee each pose their own unique problems. This study shows that utility of postural steadiness and dynamic stability could potentially be considered a useful clinical tool to measure quantifiable outcomes following acute injuries. 
Author Contributions: Conceptualization, K.G., M.S., T.T. and J.W.; methodology, K.G., M.S., T.T. and J.W.; software, T.T. and J.W.; validation, K.G., M.S., T.T. and J.W.; formal analysis, M.S., T.T., and J.W.; investigation, K.G., M.S., T.T. and J.W.; resources, K.G.; data curation, M.S., T.T., and J.W.; writing-original draft preparation, K.G., M.S., T.T. and J.W.; writing-review and editing, K.G.; visualization, K.G., M.S., T.T. and J.W.; supervision, T.L.; project administration, T.L.; funding acquisition, N/A. All authors have read and agreed to the published version of the manuscript.

Funding: This research received no external funding.

Institutional Review Board Statement: Ethical review and approval were waived for this study due to nature of study design as a case report.

Informed Consent Statement: Written informed consent has been obtained from the patient to publish this paper.

Data Availability Statement: Not applicable.

Conflicts of Interest: The authors declare no conflict of interest.

\section{References}

1. Phisitkul, P.; James, S.L.; Wolf, B.R.; Amendola, A. MCL injuries of the knee: Current concepts review. Iowa Orthop. J. 2006, 26, 77-90. [PubMed]

2. Duffy, P.S.; Miyamoto, R.G. Management of Medial Collateral Ligament Injuries in the Knee: An Update and Review. Physician Sportsmed. 2010, 38, 48-54. [CrossRef]

3. Mordecai, S.C.; Al-Hadithy, N.; E Ware, H.; Gupte, C.M. Treatment of meniscal tears: An evidence based approach. World J. Orthop. 2014, 5, 233-241. [CrossRef] [PubMed]

4. Wijdicks, C.A.; Griffith, C.J.; Johansen, S.; Engebretsen, L.; Laprade, R.F. Injuries to the Medial Collateral Ligament and Associated Medial Structures of the Knee. J. Bone Jt. Surg. Am. Vol. 2010, 92, 1266-1280. [CrossRef] [PubMed]

5. Del Din, S.; Hickey, A.; Ladha, C.; Stuart, S.; Bourke, A.K.; Esser, P.; Rochester, L.; Godfrey, A.; Stuart, S. Instrumented gait assessment with a single wearable: An introductory tutorial. F1000Research 2016, 5, 2323. [CrossRef]

6. Soangra, R.; Lockhart, T.E. Inertial Sensor-Based Variables Are Indicators of Frailty and Adverse Post-Operative Outcomes in Cardiovascular Disease Patients. Sensors 2018, 18, 1792. [CrossRef] [PubMed]

7. Paulich, M.; Schepers, M.; Rudigkeit, N.; Bellusci, G. Xsens MTw Awinda: Miniature Wireless Inertial-Magnetic Mo-tion Tracker for Highly Accurate 3D Kinematic Applications [White paper] Xsens Technologies B.V. Available online: https: / www.xsens. com/hubfs/3446270/Downloads/Manuals/MTwAwinda_WhitePaper.pdf (accessed on 6 April 2017).

8. Hauger, A.V.; Reiman, M.P.; Bjordal, J.M.; Sheets, C.; Ledbetter, L.; Goode, A.P. Neuromuscular electrical stimulation is effective in strengthening the quadriceps muscle after anterior cruciate ligament surgery. Knee Surg. Sports Traumatol. Arthrosc. 2017, 26, 399-410. [CrossRef] [PubMed]

9. Tjernström, F.; Björklund, M.; Malmström, E.M. Gait \& Posture Romberg ratio in quiet stance posturography-Test to retest reliability. Gait Posture 2015, 42, 27-31. [PubMed]

10. Lockhart, T.E.; Liu, J. Differentiating fall-prone and healthy adults using local dynamic stability. Ergonomics 2008, 51, 1860-1872. [CrossRef] [PubMed]

11. Hak, L.; Houdijk, J.; Beek, P.J.; Van Dieën, J.H. Steps to Take to Enhance Gait Stability: The Effect of Stride Frequency, Stride Length, and Walking Speed on Local Dynamic Stability and Margins of Stability. PLoS ONE 2013, 8, e82842. [CrossRef] [PubMed]

12. Van Der List, J.P.; DiFelice, G.S. Primary Repair of the Medial Collateral Ligament with Internal Bracing. Arthrosc. Tech. 2017, 6, e933-e937. [CrossRef] [PubMed]

13. Varelas, A.N.; Erickson, B.J.; Cvetanovich, G.L.; Bach, B.R. Medial Collateral Ligament Reconstruction in Patients with Medial Knee Instability: A Systematic Review. Orthop. J. Sports Med. 2017, 5. [CrossRef] [PubMed] 